\title{
XMM-Newton observations of RR Telescopii: evidence for wind signatures and shocked gas emission
}

\author{
R. González-Riestra ${ }^{1}$, P. Selvelli ${ }^{2}$, and A. Cassatella ${ }^{3,4}$ \\ 1 XMM-Newton Science Operations Centre, ESAC, 28691 Madrid, Spain \\ e-mail: Rosario.Gonzalez@sciops . esa.int \\ 2 INAF - Osservatorio Astronomico di Trieste, 34143 Trieste, Italy \\ 3 Departamento de Física Teórica, Universidad Autónoma de Madrid, 28049 Madrid, Spain \\ 4 INAF - IAPS, Via del Fosso del Cavaliere 100, Rome, Italy \\ Received 25 February 2013 / Accepted 14 June 2013
}

\begin{abstract}
RR Telescopii is the prototype of the symbiotic novae class. Since its outburst in 1944, it has been slowly fading, and its emission-line spectrum has evolved significantly. In this paper we discuss XMM-Newton observations of RR Tel taken in April 2009. These are the first X-ray high-resolution spectra of this system, and they provide important information about the physical conditions of the emitting gas. We have estimated the temperature and the luminosity of the hot star to be $154 \mathrm{kK}$ and $5000 L_{\odot}$, respectively, from the comparison of model atmospheres with the X-ray spectra. Normalisation of the models with the far ultraviolet flux leads to similar values. Both the shape of the low-resolution X-ray spectrum and the spectral diagnostics that make use of the emission lines present in the RGS spectrum indicate the existence of collisionally ionised gas. At least two components are present, with temperatures of $\approx 0.6$ and 1.7 MK. The existence of a wind from the hot star is unequivocally confirmed by HST-STIS observations taken in 2000, which showed displaced absorption components for the N V $1240 \AA$ and C IV $1550 \AA$ A doublets. These components have terminal velocities of the order of $400 \mathrm{~km} \mathrm{~s}^{-1}$, which is consistent with the temperature of the gas detected in X-rays.
\end{abstract}

Key words. binaries: symbiotic - X-rays: binaries - stars: individual: RR Telescopii

\section{Introduction}

RR Telescopii is one of the best-studied symbiotic stars. It is the prototype of the symbiotic novae class and, within this group, is the one showing the slowest evolution. Its outburst started in October 1944, when its visual magnitude was $\approx 14$, and reached its maximum brightness at magnitude 7 more than one year later. It stayed at maximum during five years and then started to turn down. Nearly 70 years after the outburst, the system is still declining and is currently at visual magnitude $\approx 12$ (from AAVSO lightcurve).

Already before the 1944 outburst, RR Tel was known to be a Mira variable (spectral type M5 III) with a period of 387 days. Based on this period, Whitelock (1988) estimated a distance to RR Tel of $2.6 \mathrm{kpc}$. This is the value commonly adopted. Nevertheless, there are indications that the absorption could have been overestimated in the past. Selvelli et al. (2007) used the intensity ratios of the He II Fowler lines to derive the extinction, obtaining a value of $E(B-V) \approx 0.00$, while values previously adopted were in the range $0.08-0.10$. This low reddening would place the system at a distance of $3.5 \mathrm{kpc}$.

The binary nature of RR Tel and the presence of a hot white dwarf in the system were established from the first International Ultraviolet Explorer (IUE) observations in the late '70s (Selvelli et al. 1979). This hot star ionised the extended envelope of the Mira, giving rise to a large number of emission lines in the optical and ultraviolet (UV) ranges. The orbital period of the system and the distance between the two stars both remain unknown at present.
The optical and UV spectrum of the system is extraordinarily rich, with emission lines spanning a wide range in ionisation and excitation, and has undergone remarkable changes over the years. Examples can be seen in Selvelli \& Bonifacio (2000) and Selvelli et al. (2007). Penston et al. (1983) made a thorough study of the IUE spectrum of RR Tel, where they identified more than 400 emission lines in the UV range. Harper et al. (1999) used HST Goddard High Resolution Spectrograph (GHRS) spectra to provide constraints to atomic data for O IV. More recently, using Space Telescope Imaging Spectrograph (STIS) and ESO Ultraviolet and Visual Echelle Spectrograph (UVES) spectra, Young et al. (2011) derived wavelengths for a large set of forbidden and intercombination lines, and Young (2012) identified up to nineteen O VI recombination lines.

Einstein and EXOSAT made the first detection of RR Tel in X-rays. Kwok \& Lehay (1984) interpreted the Einstein observations as bremstrahlung emission from a gas with a temperature of $5 \mathrm{MK}$. The mechanism responsible for this high-temperature gas was ascribed to wind-wind interaction, i.e. the collision and consequent shock between the fast wind of the nova and the slow wind of the M5 III star.

The ROSAT observation of RR Tel obtained in 1992 was analysed in detail by Jordan et al. (1994). The PSPC spectrum is dominated by a super-soft component. A fit to model atmospheres gave an effective temperature of $142 \mathrm{kK}$ and a luminosity of $3500 L_{\odot}$. These authors describe the presence of a harder, much weaker component, which is too faint to make a detailed analysis; they also speculated that it could be originated in shocks between the winds of the two stars. 
Table 1. Summary of XMM-Newton X-ray observations of RR Tel.

\begin{tabular}{lcccc}
\hline \hline Instrument & Mode/Filter & Start $^{a}$ & $\mathrm{~T}_{\exp }{ }^{b}$ & Count rate $^{c}$ \\
\hline pn & Full frame/Thin & $17: 07$ & 67 & $226 \pm 2$ \\
MOS1 & Full frame/Thin & $16: 45$ & 84 & $22 \pm 1$ \\
MOS2 & Full frame/Medium & $16: 45$ & 87 & $20 \pm 1$ \\
RGS1 & Spectroscopy & $15: 56$ & 88 & $4 \pm 1$ \\
RGS2 & Spectroscopy & $15: 56$ & 88 & $3 \pm 1$ \\
\hline
\end{tabular}

Notes. ${ }^{(a)}$ Start time is UT of 2009-04-14. ${ }^{(b)} T_{\text {exp }}$ : effective exposure time (in ks) after removal of high background periods. ${ }^{(c)}$ Count rates (in $10^{-3} \mathrm{cts} \mathrm{s}^{-1}$ ) in the range $0.2-2 \mathrm{keV}$ for EPIC and 6-38 A for RGS.

Swift observations taken in 2007 confirmed the presence of the soft component at a flux level similar to that detected by ROSAT 15 years before (Ness et al. 2007).

In this paper we describe the observations of RR Tel obtained in 2009 with XMM-Newton. A preliminary analysis of these data was presented in González-Riestra et al. (2012). We now complement these observations with STIS spectra taken in 2000.

\section{XMM observations and data reduction}

RR Tel was observed in April 2009 (obsid 05511501) by all the instruments on-board XMM-Newton, including the Optical Monitor (OM). Details of the exposures taken with the X-ray instruments are summarised in Table 1, and a similar summary for OM is found in Table 3.

The XMM-Newton data have been reduced with the most recent version of the Science Analysis System (SAS 12.1) and with the most up-to-date calibration data as of September 2012. The observations were partly affected by background flares. These periods of high radiation were removed before analysing the data. Spectra from the EPIC-MOS cameras were extracted from a circular region of about 40 arcsec of radius around the target. For the three cameras, the background was extracted from a closeby circular region. The distribution of event patterns in the EPIC-pn data was found to be substantially different from the expected one, and the core of the point spread function was excised to minimise the effect of pile-up in the spectral analysis. The RGS and OM data were processed in a standard way. We discuss EPIC data in Sect. 3, RGS spectra in Sect. 4, and OM observations in Sect. 5.

\section{The XMM-Newton EPIC spectra}

Table 1 gives the observed count rates in the three EPIC cameras in the range 0.2-2.0 keV. The spectra are shown in Fig. 1.

We attempted to model the XMM-Newton EPIC data with XSPEC. The fit was performed on spectra rebinned in order to oversample the full width half maximum of the energy resolution by a factor 3 and to have a minimum of 25 counts per bin. In addition, the spectral range was restricted to $0.2-2 \mathrm{keV}$ for the three cameras, as the signal is extremely low at higher energies.

To account for the emission of the hot star, we used the nonlocal thermodynamic equilibrium (NLTE) stellar atmosphere models for hot stars described by Rauch $(2003)^{1}$. This web provides grids of NLTE stellar model fluxes for hot stars with temperatures between 50 and $190 \mathrm{kK}$ and surface gravities in the range $\log g=5-9$. In this case we have selected models with solar abundance and $\log g=6$.

\footnotetext{
1 http://astro.uni-tuebingen.de/ rauch/TMAF/TMAF .html
}

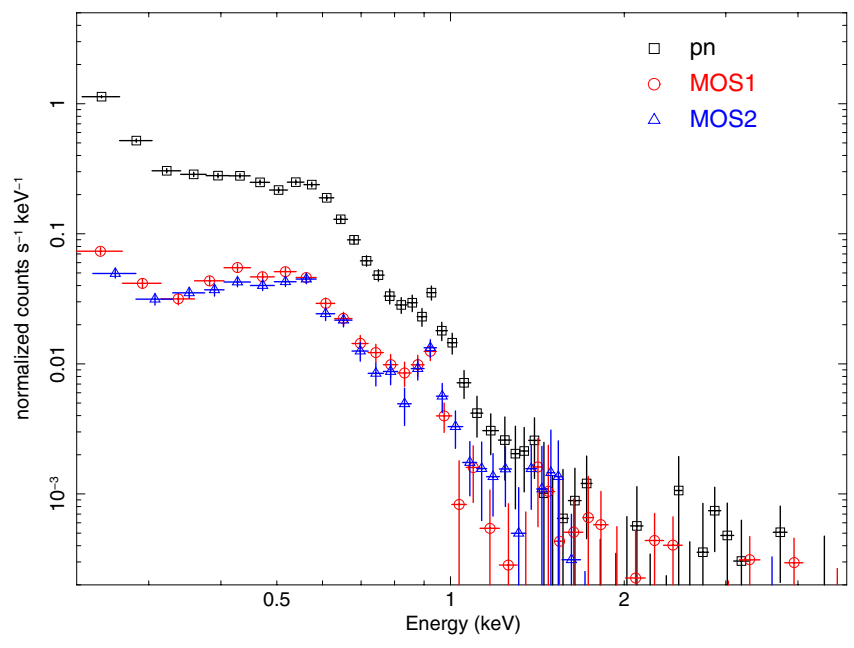

Fig. 1. XMM-Newton EPIC spectra of RR Tel. For clarity, the spectra shown here have been rebinned in order to oversample the full width half maximum of the energy resolution by a factor 3 and to have a minimum of ten counts per bin.

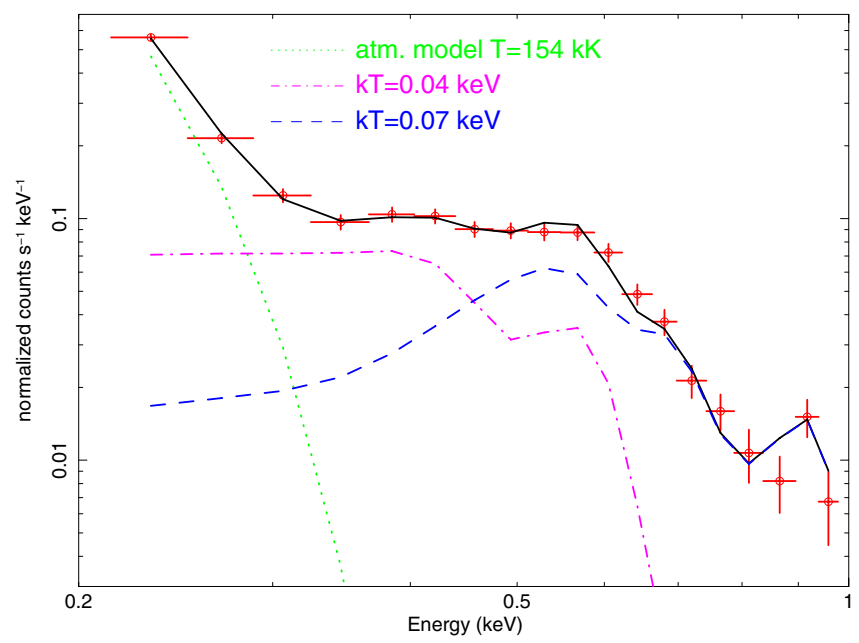

Fig. 2. Detail of XMM-Newton EPIC-pn spectrum of RR Tel showing the fit to the atmosphere model and the two MEKAL plasmas. Though the fit has been performed using the data from the three EPIC cameras, only EPIC-pn is shown for clarity.

The flux excess observed above $\approx 0.4 \mathrm{keV}$ (see Fig. 2) indicates clearly that, in addition to stellar emission, a contribution from gas emission is present that should be suitably taken into account.

Nevertheless, we found that a single-temperature gas cannot reproduce the overall continuum shape of the spectrum. We then used a model with three components: the aforementioned atmosphere models, and two MEKAL plasmas which have different temperatures and are affected by different amounts of absorption (Fig. 2).

The derivation of accurate parameters from the atmosphere models is made more difficult by the limited response of the EPIC camera in the soft energy range. For this reason we had to constrain some of the parameters of the fit beforehand. In first place, based on the values of the colour excess $E(B-V)$ as derived from various authors (see Table 4 ), we could set an upper limit to the hydrogen column density of $6 \times 10^{20} \mathrm{~cm}^{-2}$. Based on the values presented in the same table, the lower limit to the effective temperature of the hot star was set to $130 \mathrm{kK}$. 

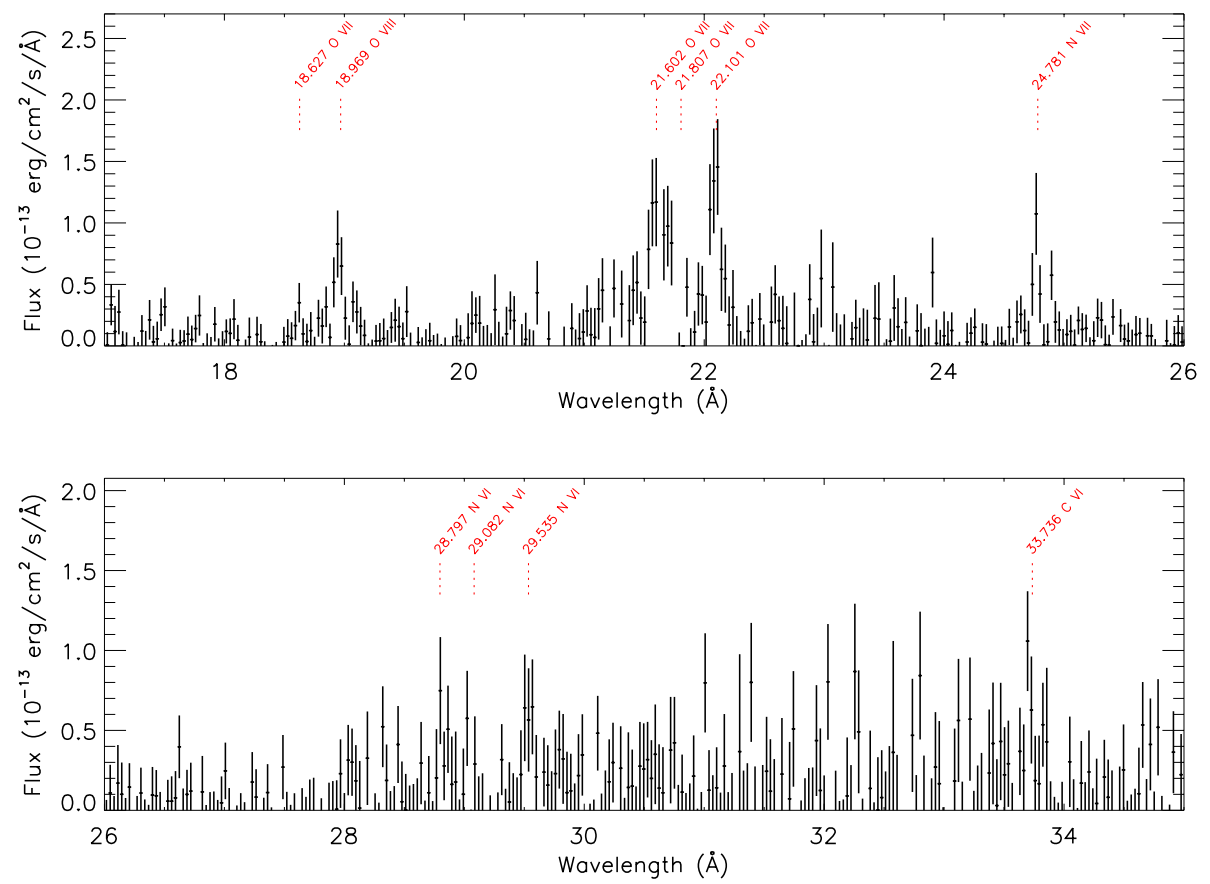

Fig. 3. RGS fluxed spectrum of RR Tel. This spectrum is a combination of RGS1 and RGS2 data. The labels mark the strongest lines.
The best-fit value of the temperature of the hot star is $154 \mathrm{kK}$, which implies (assuming a distance of $2.6 \mathrm{kpc}$ ) a luminosity of $5000 L_{\odot}$. The best-fit column density is $2.8 \times 10^{20} \mathrm{~cm}^{-2}$ (equivalent to $E(B-V)=0.047$ for a standard gas-to-dust ratio of $6 \times 10^{21} \mathrm{~cm}^{-2} \mathrm{mag}^{-1}$ (Bohlin et al. 1978)). We recall that Jordan et al. (1994) obtained values of $N_{\mathrm{H}}$ in the range $1.7-3.1 \times 10^{20} \mathrm{~cm}^{-2}$ from ROSAT PSPC observations.

The temperatures of the two plasmas are 0.04 and $0.07 \mathrm{keV}$ $(\approx 0.5$ and $0.8 \mathrm{MK})$. The first one is the main contributor below $0.4 \mathrm{keV}$. The second one is required to reproduce the peaks around 0.6 and $0.9 \mathrm{keV}$, which correspond to the He-like triplets of O VII and Ne IX (see Fig. 2). These two plasmas are affected by additional absorption, with hydrogen columns densities of 1.7 and $6.4 \times 10^{21} \mathrm{~cm}^{-2}$, respectively, both at least ten times higher than the interstellar column density. The emission measure of the two plasmas is $2 \times 10^{59}$ and $5 \times 10^{58} \mathrm{~cm}^{3}$, respectively, for a distance of $2.6 \mathrm{kpc}$, and their luminosities, correcting only for interstellar absorption, are 0.04 and $0.02 L_{\odot}$, respectively, in agreement with the estimation made by Jordan et al. (1994). Taking into account the absorption that affects each of the components, their intrinsic luminosities in the range $0.2-10 \mathrm{keV}$ are approximately 20 and $70 L_{\odot}$, respectively.

\section{The RGS spectrum}

The RGS spectrum of RR Tel is weak, with a count rate of $0.004-0.003 \mathrm{cts} \mathrm{s}^{-1}$ for RGS1 and RGS2, respectively. The difference in count rate between the two instruments reflects the fact that the main contributor to the overall flux is the O VII Helike triplet, which only appears in RGS1. No continuum flux is detected. The model derived from the EPIC data described above predicts a flux at $20 \AA$ of $\approx 1 \times 10^{-15} \mathrm{erg} \mathrm{cm}^{-2} \mathrm{~s}^{-1} \AA^{-1}$, equivalent to $6 \times 10^{-5} \mathrm{RGS}$ cts s$^{-1} \AA^{-1}$, which is in good agreement with the observed $3 \sigma$ upper limit of $5 \times 10^{-3} \mathrm{cts} \mathrm{s}^{-1} \AA^{-1}$. The strongest lines present in the spectrum, apart from the O VII triplet, are the Lyman $\alpha$ lines of O VIII, N VII, and C VI, along with the $\mathrm{N}$ VI triplet (Fig. 3). Their wavelengths and intensities, the latter obtained through a fit with XSPEC, are given in Table 2. Due to the low number of counts per bin, the C-statistic has been used
Table 2. Emission lines in the RGS spectrum of RR Tel.

\begin{tabular}{clcc}
\hline \hline $\begin{array}{c}\text { Wavelength } \\
\AA\end{array}$ & Ion & $\begin{array}{c}\text { Observed Flux } \\
10^{-14} \mathrm{erg} \mathrm{cm}^{-2} \mathrm{~s}^{-1}\end{array}$ & C/d.o.f. $^{a}$ \\
\hline 18.63 & O VII & $<0.36$ & $257 / 209$ \\
18.97 & O VIII & $1.05+/-0.40$ & $\prime \prime$ \\
21.60 & O VII $(r)$ & $2.27+/-0.78$ & $164 / 154$ \\
21.80 & O VII $(i)$ & $<0.36$ & $\prime \prime$ \\
22.09 & O VII $(f)$ & $1.91+/-0.49$ & $\prime \prime$ \\
24.78 & N VII & $1.19+/-0.44$ & $301 / 282$ \\
28.78 & N VI $(r)$ & $1.32+/-0.73$ & $477 / 376$ \\
29.08 & N VI $(i)$ & $<0.41$ & $\prime \prime$ \\
29.54 & N VI $(f)$ & $1.21+/-0.48$ & $\prime \prime$ \\
33.73 & C VI & $0.94+/-0.45$ & $112 / 98$ \\
\hline
\end{tabular}

Notes. ${ }^{(a)} \mathrm{C}$ : value of the C-statistic, d.o.f.: degrees of freedom

Diagnostics.

\begin{tabular}{lccccc}
\hline \hline & $G^{a}$ & $R^{b}$ & $R_{\text {ion }}{ }^{c}$ & $\begin{array}{c}T_{\mathrm{e}} \\
\mathrm{MK}\end{array}$ & $\begin{array}{c}N_{\mathrm{e}} \\
\mathrm{cm}^{-3}\end{array}$ \\
\hline Oxygen & $0.8-1.0$ & $>3.7$ & 0.5 & $1.4-2.1$ & $<7 \times 10^{8}$ \\
Nitrogen & $0.9-1.2$ & $>2.0$ & 0.9 & $0.5-1.0$ & $<8 \times 10^{9}$ \\
\hline
\end{tabular}

Notes. ${ }^{(a)} G=(i+f) / r .{ }^{(b)} R=f / i .{ }^{(c)} R_{\text {ion }}$ : ratio of H-like and He-like resonance lines.

for the fits. The background has not been subtracted but modelled together with the lines, which have been assumed to be Gaussian functions. None of the lines shows evidence for intrinsic broadening.

The He-like triplets are a powerful tool for the diagnostics of the ionisation processes in the plasma (see Porquet \& Dubau 2000; Porquet et al. 2001, 2010). The "R" ratio (ratio of the intensities of the forbidden and the intercombination lines) is sensitive to the electron density, and the "G" ratio (ratio of the intensities of the forbidden plus the intercombination lines and the resonance line) is sensitive to the electron temperature. In a purely photoionised plasma, the value of $\mathrm{G}$ is always larger than four. A strong resonance line (i.e. a low value of $G$ ) is indicative 
Table 3. Summary of XMM-Newton OM observations of RR Tel.

\begin{tabular}{|c|c|c|c|c|}
\hline Filter & $\begin{array}{l}\text { Start } \\
\text { UT }\end{array}$ & $\begin{array}{c}\text { Duration } \\
\mathrm{s}\end{array}$ & Count rate & Flux $^{a}$ \\
\hline$U V W 1$ & 2009-04-14 18:48 & 4400 & $443 \pm 2$ & 2.11 \\
\hline$U V W 1$ & 2009-04-14 20:07 & 4400 & $440 \pm 7$ & 2.10 \\
\hline UVW1 & 2009-04-14 21:25 & 4400 & $443 \pm 2$ & 2.11 \\
\hline$U V W 1$ & 2009-04-14 23:14 & 4400 & $442 \pm 9$ & 2.10 \\
\hline$U V W 1$ & 2009-04-15 00:33 & 4400 & $443 \pm 2$ & 2.11 \\
\hline UVM2 & 2009-04-15 01:51 & 4400 & $70.6 \pm 0.1$ & 1.55 \\
\hline$U V M 2$ & 2009-04-15 07:06 & 4400 & $70.0 \pm 0.1$ & 1.55 \\
\hline$U V W 2$ & 2009-04-15 08:25 & 4400 & $33.7 \pm 0.1$ & 1.93 \\
\hline$U V W 2$ & 2009-04-15 13:40 & 4400 & $33.6 \pm 0.1$ & 1.92 \\
\hline$U V W 2$ & 2009-04-15 14:58 & 4300 & $33.6 \pm 0.1$ & 1.92 \\
\hline$U V W 2$ & 2009-04-15 16:16 & 4300 & $34.6 \pm 0.1$ & 1.95 \\
\hline$U V W 2$ & 2009-04-15 17:33 & 4300 & $33.4 \pm 0.1$ & 1.91 \\
\hline$U V W 2$ & 2009-04-15 18:50 & 4300 & $34.0 \pm 0.1$ & 1.94 \\
\hline$U V W 2$ & 2009-04-15 20:07 & 4300 & $33.5 \pm 0.1$ & 1.91 \\
\hline
\end{tabular}

Notes. ${ }^{(a)}$ Observed flux in units of $10^{-13} \mathrm{erg} \mathrm{cm}^{-2} \mathrm{~s}^{-1} \AA^{-1}$.

of the importance of collisional processes. A low value of $R$ indicates a high electron density.

The intensity of the resonance line in the two He-like triplets detected in the RGS spectrum of RR Tel is comparable to that of the forbidden line (Fig. 4). This is a clear indication of the importance of collisional processes in the plasma (Porquet et al. 2010). On the other hand, the intercombination line is extremely faint in both cases, so that only an upper limit to its intensity can be given.

The value of the $\mathrm{G}$ parameter is $\approx 1.0$ for the O VII triplet, and 1.2 for the N VI triplet. Using the latest version of the CHIANTI database (v7.0, Landi et al. 2012), the corresponding electron temperatures are $1.4 \times 10^{6} \mathrm{~K}$ for O VII and $5.0 \times 10^{5} \mathrm{~K}$ for $\mathrm{N}$ VI. The temperatures at maximum ionisation ratio for both ions are $9 \times 10^{5} \mathrm{~K}$ and $6 \times 10^{5} \mathrm{~K}$. These two temperatures are in good agreement with those derived from the fit to the EPIC spectra.

Only a lower limit can be computed for $\mathrm{R}$ in both triplets, being $>3.7$ and $>2.0$ for O VII and N VI, respectively, which indicates that they are formed in a low-density region. Upper limits to the electron density are $7 \times 10^{8} \mathrm{~cm}^{-3}$ for O VII and $8 \times 10^{9} \mathrm{~cm}^{-3}$ for N VI.

This result provides unambiguous evidence for the coexistence in RR Tel of a collisionally ionised plasma with the well-established low-density photoionised nebula (Hayes \& Nussbaumer 1996; Young 2012).

\section{The OM UV data}

RR Tel was extensively monitored with the IUE satellite during the period 1978-1995 (see e.g. Hayes \& Nussbaumer 1996; Mürset \& Nussbaumer 1994). For reference, IUE fluxes in 1995 were $1.98,1.22$, and $2.16 \times 10^{-13} \mathrm{erg} \mathrm{cm}^{-2} \mathrm{~s}^{-1} \AA^{-1}$ at the effective wavelengths of the $U V W 2, U V M 2$, and $U V W 1$ filters, respectively.

$X M M-N e w t o n$ OM exposures were performed in parallel with the X-ray observations. The filters used were $U$ (effective wavelength $3440 \AA), U V W 1$ (2910 ̊), UVM2 (2310 ̊) and UVW2 (2120 ̊). Details about the start time and duration of the exposures in each filter are given in Table 3 . In all cases the $\mathrm{OM}$ instrumental configuration was such that it allowed, apart
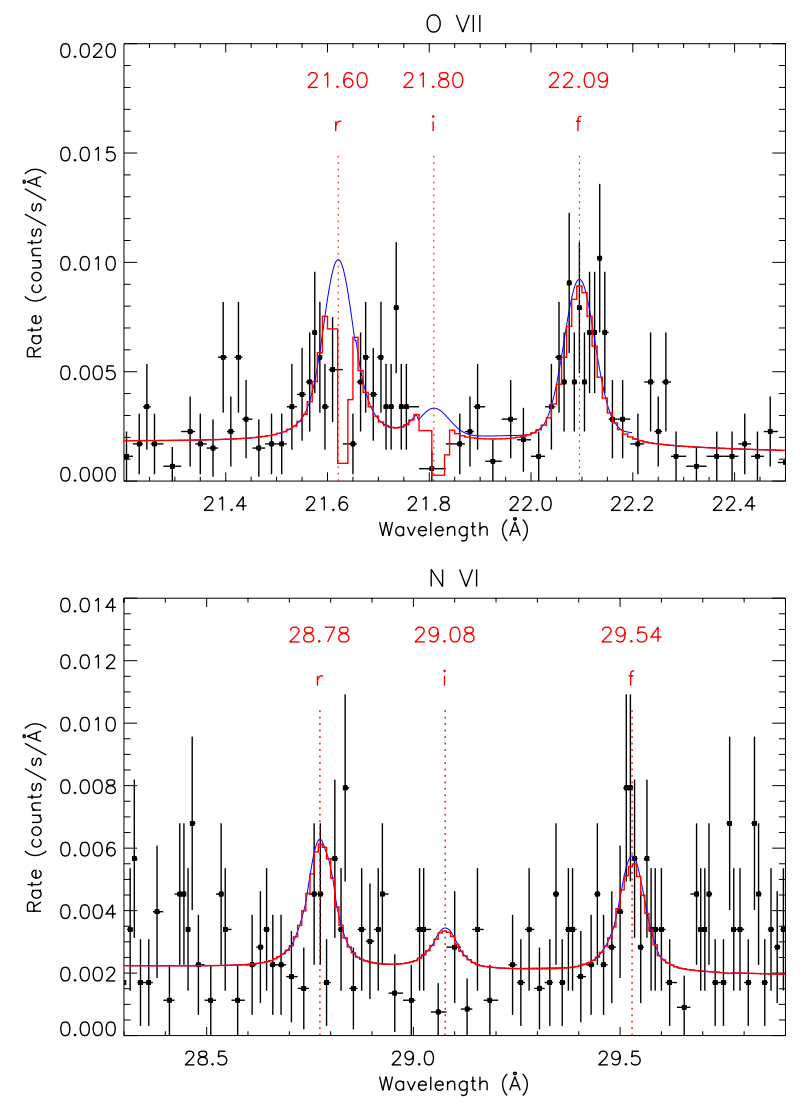

Fig. 4. O VII (RGS1) and N VI (RGS2) He-like multiplets in RR Tel. The spectra have been binned to a minimum of three counts per bin. The red line shows the best-fit model for the resonance and forbidden lines. The intercombination line is shown with its intensity set to the upper obtained from the fit. Dips due to bad columns are clearly seen near 21.6 and $21.8 \AA$. The blue line in the top panel shows the analytical representation of the three Gaussians, broadened with the instrumental width ( $\approx 0.07 \AA$ FWHM) and having the intensities derived in the fit.

form the imaging window, a small window in the centre of the field in fast mode, with a time resolution of $0.5 \mathrm{~s}$. RR Tel was detected in all the four OM filters. Due to the brightness of the target, the coincidence loss corrections to the $U$ and to some of the $U V W 1$ observations are highly uncertain, and therefore these data are less reliable. Some of the exposures were lost due to instrumental problems. The fluxes obtained from the OM photometric measurements must be taken only as indicative, since the conversion from count rate to flux is made assuming a white dwarf-like spectral shape, and in the case of RR Tel the contribution of the nebular continuum and the emission lines is not negligible.

To make a more accurate comparison, we folded the IUE spectra through the OM filters response and we estimated the equivalent fluxes in the OM bands. The result is shown in Fig. 5. For the three OM UV filters, the 2009 value agrees nicely with the extrapolation of the IUE fluxes. The lines shown in the figure correspond to the fit of the IUE fluxes to an exponential function. The e-folding times are of the order of 23 years.

The high time resolution light curves obtained through all the filters show some degree of variability. There is not any evident correlation between the UV and the X-ray light curves. This is not surprising, because at the wavelengths sampled by the OM UV filters the dominant contributor to the spectrum is the Balmer recombination continuum, while in the range $0.2-2 \mathrm{keV}$, 
Table 4. Determinations of temperature and radius of RR Tel.

\begin{tabular}{lccccl}
\hline \hline Year & $\begin{array}{c}\text { Temperature } \\
\mathrm{kK}\end{array}$ & $\begin{array}{c}\text { Radius } \\
R_{\odot}\end{array}$ & $\begin{array}{c}\text { Luminosity } \\
L_{\odot}\end{array}$ & $E(B-V)$ & Reference \\
\hline $1978-1984$ & $150-200$ & 0.07 & $2200-6900$ & 0.09 & IUE, Hayes \& Nussbaumer (1996) \\
1978 & 135 & 0.17 & 8800 & 0.10 & IUE, Mürset et al. (1991) \\
1983 & 140 & 0.12 & 4900 & 0.10 & IUE, Mürset et al. (1991) \\
1992 & 140 & 0.09 & 2700 & 0.10 & IUE, Mürset \& Nussbaumer (1994) \\
$1992^{a}$ & 142 & 0.09 & 3500 & 0.03 & IUE+VOYAGER+ROSAT, Jordan et al. (1994) \\
1992 & 135 & 0.09 & 2500 & 0.06 & IUE+VOYAGER+ROSAT, Jordan et al. (1994) \\
1995 & 140 & 0.11 & 3700 & 0.08 & HST+ORFEUS+HUT, Nussbaumer \& Dumm (1997) \\
$2009^{a}$ & 154 & 0.10 & 5000 & 0.06 & This work \\
\hline
\end{tabular}

Notes. Distance is in all cases assumed to be $2.6 \mathrm{kpc}$, except in the first one, where it is assumed to be $3.6 \mathrm{kpc}$ (a) $^{(a)}$ Temperature and extinction from fit to model atmosphere. Otherwise they are derived from fits to a blackbody.

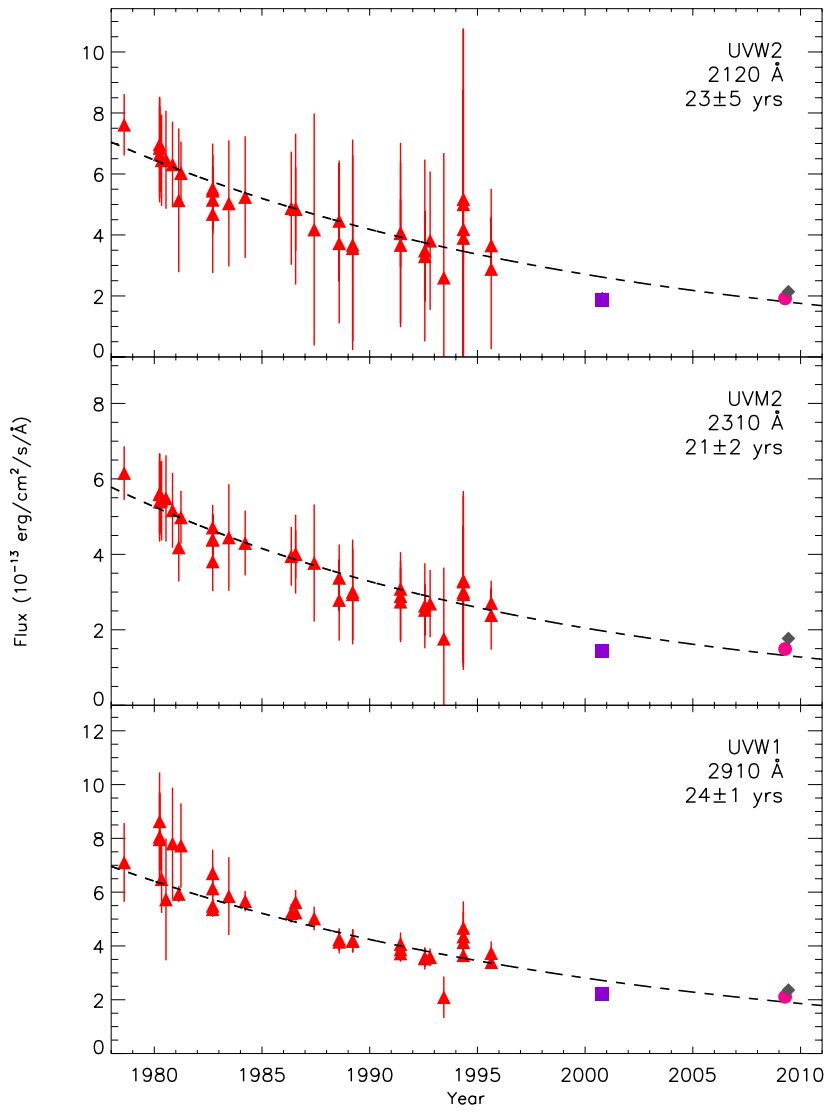

Fig. 5. Evolution of the UV flux of RR Tel. Triangles are fluxes in the $X M M$-Newton OM bands computed from IUE spectra. Filled circles are fluxes measured by the XMM-Newton OM. Squares and diamonds are points derived from the STIS spectrum taken in October 2000 and the GALEX spectrum taken in June 2009, respectively. We note that data taken with the $U V W 1$ filter are uncertain, as they may be affected by coincidence losses. The lines show exponential fits to the IUE data.

the spectrum is dominated by the emission from the hot star (see Fig. 6).

\section{The spectral energy distribution of RR Tel}

The spectral energy distribution of RR Tel from 5 to $3200 \AA$. is shown in Fig. 6. In order of increasing wavelength, the data shown are:

- 5-70 A: XMM EPIC-pn "fluxed" spectrum (computed with the task efluxer of the XMM SAS), which is obtained from the count spectrum, taking into account the appropriate corrections for the energy redistribution and the effective area of the telescope, filter, and detector combination. This flux is model independent.

- 900-1200 A: ORFEUS data taken in November 1996 (Krautter et al. 1998).

- 1200-3200 Å: IUE spectra taken in August 1995.

- XMM-Newton OM fluxes at 2120 and $2310 \AA$.

All the data have been corrected for interstellar absorption assuming $N_{\mathrm{H}}=2.8 \times 10^{20} \mathrm{~cm}^{-2}$ (see Sect. 3).

Also shown in the figure is a sample of the NLTE stellar atmosphere models for hot stars mentioned in Sect. 3, normalised to the ORFEUS flux at $1100 \AA$, where the contribution of the recombination continuum is still negligible.

This figure does not show a formal fit, but it is just a qualitative representation of the spectral energy distribution of the system. Nevertheless, it provides some useful information:

- The main contributor to the overall emission of the system in the X-ray/UV domain is the hot star, which dominates in the range $\approx 50-1200 \AA$.

- At shorter wavelengths ( hortward $\approx 30 \AA$ ), there is an excess flux that peaks at $\approx 20 \AA$. This emission was already detected by ROSAT (Jordan et al. 1994) and by Einstein (Kwok \& Lehay 1984), though the low sensitivity of the instrument did not allow the authors to make any detailed analysis. As shown in the RGS spectrum (see Fig. 3), the peak seen in the EPIC-pn spectrum at this energy is due to the O VII He-like triplet.

- Longward $1200 \AA$, the contribution of the recombination continuum of the photoionised nebula starts to be important (see e.g. Nussbaumer \& Dumm 1997).

- The effective temperature of the hot star is certainly between 150 and $180 \mathrm{kK}$. Assuming a radius of $0.10 R_{\odot}$, the normalisation of the models to the UV flux implies, for effective temperatures between 150 and $170 \mathrm{kK}$, distances in the range $2.7-2.8 \mathrm{kpc}$ and luminosities between 4900 and $7400 L_{\odot}$. If, on the other hand, we assume a distance of $2.6 \mathrm{kpc}$, the resulting luminosities range from 4600 and $6900 L_{\odot}$ and the corresponding hot star radii between 0.09 and $0.10 R_{\odot}$. In particular for an effective temperature of $154 \mathrm{kK}$ (see Sect. 3) and a distance of $2.6 \mathrm{kpc}$, the bolometric luminosity is $5000 L_{\odot}$ and the radius $0.095 R_{\odot}$. 


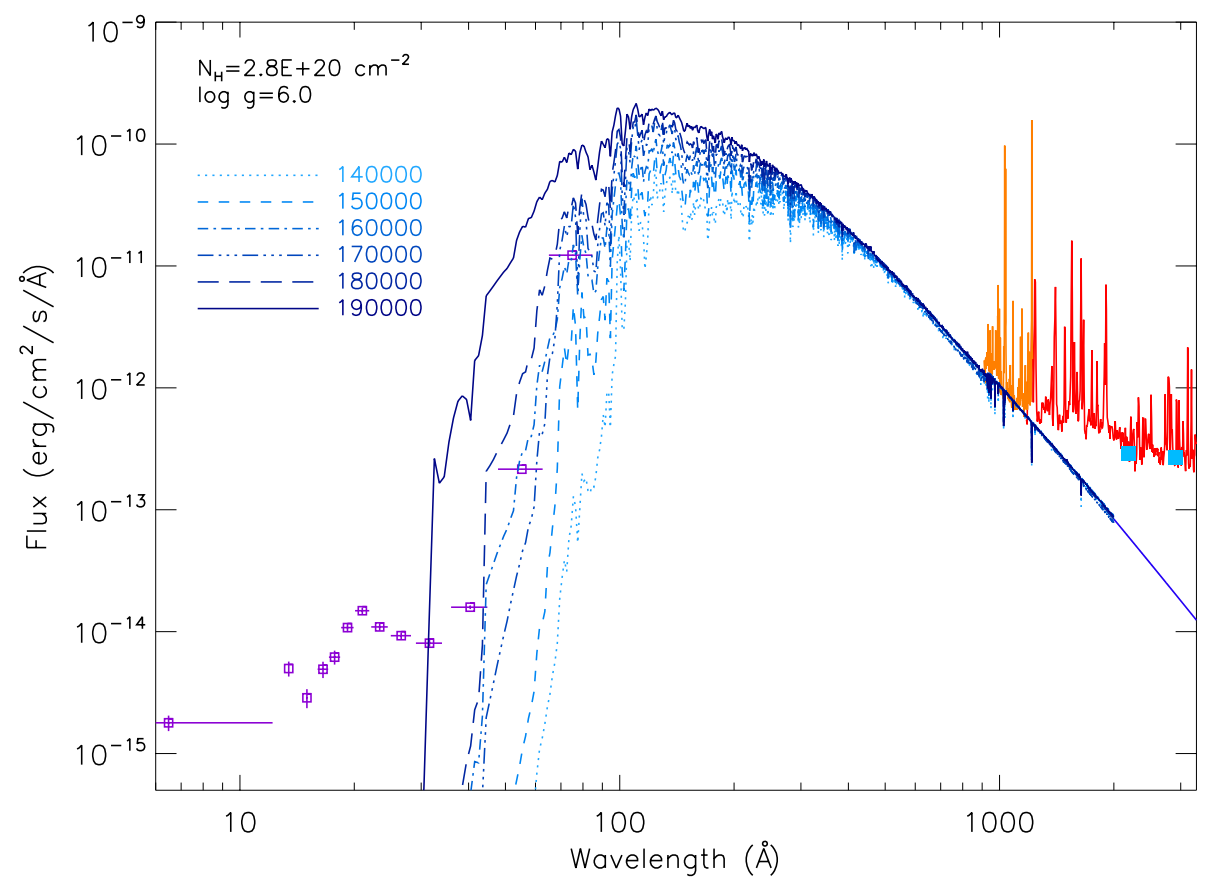

Fig. 6. Spectral energy distribution of RR Tel. The figure shows XMM-Newton EPIC, ORFEUS, and IUE data, and a set of model atmospheres normalised to the flux at $1100 \AA$ (see the text for details). All data have been corrected for an interstellar absorption equivalent to an hydrogen column density of $2.8 \times 10^{20} \mathrm{~cm}^{-2}$.

\section{Discussion}

\subsection{Consistency of the extinction derived from the $X$-ray spectrum with other determinations}

Selvelli et al. (2007) obtained a quite low hydrogen column density $\left(6.9 \times 10^{19} \mathrm{~cm}^{-2}\right)$ from the fitting of the profile of the damping wings of the interstellar Lyman $\alpha$ absorption line in a STIS high-resolution spectrum. However, an accurate re-examination of the data has revealed the presence, previously unnoticed, of a wide and shallow emission component for the Lyman $\alpha$ line. Therefore, the observed profile is essentially useless for comparison with the theoretical one. We note, however, that a lower limit to the column density can be derived from the comparison of the width of the saturated part of the profile with the theoretical one calculated for a fully damped profile. The width of the saturated profile is very sensitive to the column density and, alone, represents a good criterion for its estimate. The observed width of the saturated profile $(3.8 \AA$ ) corresponds to a lower limit of about $2.8 \times 10^{20} \mathrm{~cm}^{-2}$. This value agrees very well with the column density derived from the fit to the X-ray spectrum (see Sect. 3) and would correspond to $E(B-V) \approx 0.047$, assuming a standard gas-to-dust ratio of $6( \pm 2) \times 10^{21}$ (e.g. Bohlin et al. 1978).

However, the observed decrement of 20 recombination lines belonging to the He II Fowler series convincingly indicates that $E(B-V) \approx 0.00$, a value confirmed by the STIS continuum energy distribution in the near UV region (Selvelli et al. 2007).

This apparently contrasting evidence can be reconciled if an anomalous gas-to-dust ratio towards RR Tel is present, associated with its large distance to the galactic plane $(\geq 1.3 \mathrm{kpc})$ and/or if there is an extra contribution to $N_{\mathrm{H}}$ by a circumstellar component.

\subsection{The detection of wind signatures}

As pointed out in Sects. 1 and 3, the hard X-ray component detected by Einstein and ROSAT was attributed by Kwok \& Lehay (1984) and by Jordan et al. (1994) to bremsstrahlung emission from a high-temperature gas in the shock region, where the allegedly fast wind of the hot star and the wind of the M5 III star collide.

Hydrodynamical modelling of colliding winds in symbiotic systems predicts a warped thin shell, where the two winds collide and plasma temperatures up to $10^{7} \mathrm{~K}$ are expected (Nussbaumer \& Walder 1993; Mürset et al. 1997). Unfortunately, the predicted $\mathrm{X}$-ray emission is only an indirect evidence for wind collision, whose positive detection requires the presence of spectral features attributable to a wind, e.g. P Cygni profiles and/or violet displaced absorptions.

As extensively described by Nussbaumer \& Dumm (1997), detailed searches on IUE spectra taken from 1978 to 1992 for spectroscopic signatures of the expected fast stellar wind from the hot star gave negative results. These authors pointed out that the lack of detection was attributable to the limited dynamical range of IUE which did not allow the absorption components of very strong emission lines on top of a weak continuum to be observed. However, the same result was obtained from the examination of HST-GHRS spectra taken in 1995, whose signal in the continuum was much better. This negative result led Nussbaumer \& Dumm (1997) to conclude that there was no trace of mass-loss from 1978 onwards. Clearly, the absence was difficult to reconcile with the proposed mechanism of production of hard X-rays by colliding winds.

However, careful examination of the STIS spectrum has led us to clearly detect the presence of these long-searched displaced absorption components in both the $\mathrm{N} \mathrm{V}$ and the $\mathrm{C} \mathrm{IV}$ resonance lines (see Table 5 and Fig. 7). The displacement at the line centre corresponds to $-300 \mathrm{~km} \mathrm{~s}^{-1}$, while the terminal velocity is close to $400 \mathrm{~km} \mathrm{~s}^{-1}$, in satisfactory agreement with the $500 \mathrm{~km} \mathrm{~s}^{-1}$ estimated by Jordan et al. (1994). The lines have similar widths and similar profiles. A closer inspection of the absorption lines in the $\mathrm{N}$ V doublet, after a moderate filtering, actually shows the presence of two components separated by about $30 \mathrm{~km} \mathrm{~s}^{-1}$. These features are sharp and nearly symmetric. The residual flux at the line centre is low but not zero, close to $10 \%$ of the continuum level.

We note that the outflow is not in the form of the classical P Cygni profile as observed in early-type stars and that there is 
Table 5. Absorption lines in the STIS spectrum of RR Tel.

\begin{tabular}{lcccccccc}
\hline \hline Ion & $\lambda_{\text {lab }}$ & $\lambda_{\mathrm{em}}$ & \multicolumn{1}{c}{$\lambda_{\text {abs }}$} & $\lambda_{\infty}$ & $E W(\AA)$ & $F W H M(\AA)$ & $v\left(\mathrm{~km} \mathrm{~s}^{-1}\right)$ & $v_{\infty}\left(\mathrm{km} \mathrm{s}^{-1}\right)$ \\
\hline N V & 1238.82 & 1238.60 & 1237.39 & 1237.10 & 0.33 & 0.35 & 290 & 360 \\
N V & 1242.80 & 1242.58 & $1241.39^{a}$ & 1241.02 & 0.29 & 0.30 & 290 & 380 \\
C IV & 1548.20 & 1547.94 & $1546.40^{a}$ & 1545.92 & 0.39 & 0.48 & 300 & 390 \\
\hline
\end{tabular}

Notes. $\lambda_{\mathrm{em}}$ : Wavelength of the maximum of the emission. $\lambda_{\mathrm{abs}}$ : Wavelength of the minimum of the absorption. $\lambda_{\infty}$ : Wavelength of the absorption edge. $v=c\left(\lambda_{\mathrm{em}}-\lambda_{\mathrm{abs}}\right) / \lambda_{\mathrm{lab}} \cdot v_{\infty}=c\left(\lambda_{\mathrm{em}}-\lambda_{\infty}\right) / \lambda_{\mathrm{lab}} \cdot{ }^{(a)}$ The absorptions at 1241.39 and $1546.40 \AA$ are affected by a weak emission.
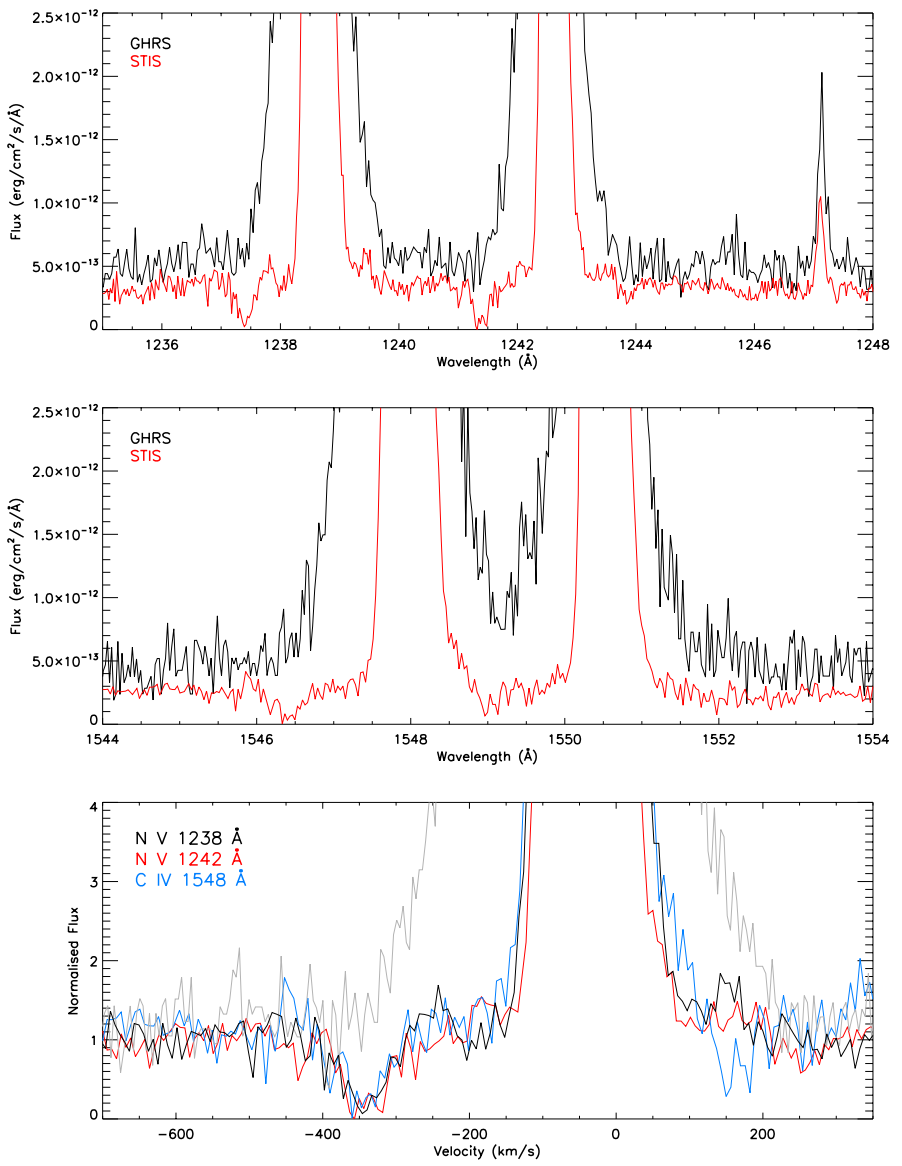

Fig.7. Upper panels: comparison of the profiles of the $\mathrm{N} V$ and C IV resonance doublets in the GHRS and STIS spectra of RR Tel taken in 1995 and 2000, respectively. The weak emission line longward the N Vdoublet is C III $1247.3 \AA$. Its width is similar in both spectra: $0.06 \AA$ in STIS and $0.07 \AA$ in GHRS. Bottom panel: comparison of the STIS profiles of the two N V lines and the $1548 \AA \mathrm{C}$ IV line. The grey line shows, for reference, the N V $1238 \AA$ line in the GHRS spectrum. The four spectra shown in this panel have been normalised to the continuum.

no physical association between the displaced absorption components and the emission components in the resonance lines of $\mathrm{C}$ IV and $\mathrm{N} \mathrm{V}$, which display a nearly symmetric profile and are of nebular origin. The absence of a typical P Cygni profile positively indicates that the wind phenomenology is far from that of a continuous outflow in a spherically symmetric, extended region.

The displaced absorption lines observed in the STIS spectrum of RR Tel are evocative of the narrow, shortward displaced absorption components (DACs) observed in the resonance lines of the UV spectra of early-type stars and ascribed to a discontinuous, inhomogeneous loss of matter, which results in "puffs" of material ejected at irregular intervals (e.g. Lamers et al. 1978; Kaper \& Henrichs 1994).

In most cases the two phenomena of $\mathrm{P}$ Cygni and DACs co-exist, but there are cases in which only the sharp displaced components are observed. In a study of the symbiotic star AG Dra, Mikolajewska et al. (1995) reported that the N V $1240 \AA$ line often displayed weak blue-shifted absorption components, suggesting a low velocity wind from the hot component at velocities of a few hundred $\mathrm{km} \mathrm{s}^{-1}$. Sekeráš \& Skopal (2012) reported the presence of a sharp absorption component in the $1032 \AA$ line in a FUSE spectrum of V1016 Cyg during a quiescent phase. This feature corresponds to an outflow with velocity of about $-250 \mathrm{~km} \mathrm{~s}^{-1}$.

The presence of DACs in the UV resonance lines and of structured absorption troughs that come and go on short, aperiodic time scales has also been observed in the winds emanating from the accretion disk of cataclysmic variables in high state (e.g. Mauche 1991; Selvelli \& Friedjung 2003). Also, Orio (2012) reported that X-ray observations of novae and recurrent novae have shown that mass seems to be lost in "parcels" or discrete shells. In the case of RR Tel, the paucity of the data (one GHRS observation showing no wind, one STIS observation showing displaced absorption components) prevents any definite statement about the origin and phenomenology of the observed DACs.

An estimate for the plasma temperature in the shock can be derived from the terminal velocity of the hot wind $v_{\text {inf }}$. Since the thermal energy per particle can not exceed the kinetic energy per particle in the wind (Mürset et al. 1997), the simple relation

$k T \approx 1 / 2 m_{\mathrm{H}} v_{\infty}^{2}$

gives $T \leq 2.9 \times 10^{6} \mathrm{~K}\left(\right.$ for $v_{\infty}=380 \mathrm{~km} \mathrm{~s}^{-1}$ ).

A crude estimate of the ionic column density in the wind can be obtained from the equivalent width of the displaced absorptions and the relation

$N \approx 1.13 \times 10^{20} \frac{E W}{\lambda^{2} \mathrm{f}_{\mathrm{ik}}}$

valid in the assumption of optically thin lines, which gives lower limits of about $1.4 \times 10^{14} \mathrm{~cm}^{-2}$ and $0.8 \times 10^{13} \mathrm{~cm}^{-2}$ for the column density of the $\mathrm{N}^{+4}$ and $\mathrm{C}^{+3}$ ions in the wind.

\subsection{RR Tel still burning}

Symbiotic novae like RR Tel represent a small subclass of the symbiotic stars (Mürset \& Nussbaumer 1994). The outburst mechanism in symbiotic novae is generally assumed to be similar to that of classical novae, i.e. a thermonuclear runaway (TNR) on the surface of a white dwarf, although it differs mainly for the slower outburst speed (Nussbaumer 1998).

In this context, RR Tel represents an extreme case of slow spectral evolution after outburst, with a timescale much 
longer than that commonly observed in classical novae. Our XMM-Newton observations have clearly demonstrated that the supersoft component still dominates the spectral energy distribution, about 65 years after the outburst of 1944. The presence of mass-loss signatures in the N V and C IV resonance lines is an independent confirmation that outflow from the white dwarf, a direct consequence of the outburst, was still going on at the time of the STIS observations. This behaviour is in contrast with that of classical novae, where the post-outburst supersoft X-ray emission, indicative of remaining H-nuclear burning, lasts a few years at maximum (see Hernanz 2012).

Mürset \& Nussbaumer (1994) attributed the difference in the outburst behaviour to differences in mass accretion rates between classical and symbiotic novae: the higher accretion rate in symbiotic novae from the wind of the giant increases compressional heating and inhibits degeneracy in the accreted shell, and this eventually would lead to a slower outburst. We point out, however, that theoretical models of nova outburst (e.g. Yaron et al. 2005) predict that the white dwarf mass is the main parameter that rules the turn-off time. In classical novae, a slow spectral evolution is generally attributed to the presence of a low-mass white dwarf in the system and/or to inefficient mass-loss mechanism after outburst.

Based on the TNR models of Yaron et al. (2005), we guess that the extreme slowness of the outburst of RR Tel should be primarily ascribed to the presence of an undermassive white dwarf $\left(\leq 0.65 M_{\odot}\right)$ in the system and not to higher accretion from the wind of the giant, as proposed by Mürset \& Nussbaumer (1994). Two well known recurrent novae, T CrB and RS Oph, have an $\mathrm{M}$ giant companion but are characterised by a very short duration of the outburst. These two objects are known to have a massive white dwarf that clearly determines the principal outburst characteristics.

If a low-mass white dwarf is indeed present in RR Tel, TNR models require the accumulation of a massive hydrogen envelope to trigger ignition, and, in the observational side, the presence of a moderate expansion velocity in the ejecta. As a matter of fact, Thackeray (1977) reported that the observed displacements in the absorption components were less than those for an "average" slow nova and considerably less than those of faster novae.

A rough calculation of the ejected mass can be obtained from the estimated radius of the nebula, $10^{15} \mathrm{~cm}$ (Hayes \& Nussbaumer 1996). This radius, together with the assumption of a homogeneous shell with electron density $N_{\mathrm{e}}=3 \times 10^{6} \mathrm{~cm}^{-3}$ in the emission-line region (Hayes \& Nussbaumer 1996), provides a lower limit of $\sim 10^{-5} M_{\odot}$ for the total mass of the ejecta.

A more accurate determination of the mass of ionised hydrogen in the nebula can be obtained from the $\mathrm{H}_{\beta}$ luminosity, $L_{\beta}=1.42 \times 10^{34} \mathrm{erg} \mathrm{s}^{-1}$, which is obtained from the $\mathrm{H}_{\beta}$ flux measured in STIS $\left(1.62 \times 10^{-11} \mathrm{erg} \mathrm{cm}^{-2} \mathrm{~s}^{-1}\right)$, assuming a distance of $2.6 \mathrm{kpc}$.

By combining the two common relations

$L_{\beta}=1.24 \times 10^{-25} N_{\mathrm{e}} \mathrm{N}^{+}(4 / 3) \pi R_{\mathrm{neb}}^{3} \epsilon$

and

$M_{\text {neb }}=\mu \mathrm{N}^{+} m_{\mathrm{H}}(4 / 3) \pi R_{\text {neb }}^{3} \epsilon$

(where $\mu=1.4$ is the mean atomic weight and $\epsilon$ is the filling factor), one finds that the hydrogen mass of the nebula (independent of $\epsilon$ and $R_{\text {neb }}$ ) is

$M_{\text {neb }}=18.67 \frac{L_{\beta}}{N_{\mathrm{e}}}$.
If $N_{\mathrm{e}} \sim 3 \times 10^{6} \mathrm{~cm}^{-3}$ (Hayes \& Nussbaumer 1996), one finally obtains that $M_{\text {neb }} \sim 4.4 \times 10^{-5} M_{\odot}$.

Using the observed intensities of the $\lambda 1640$ and $\lambda 4686$ recombination lines, similar calculations for the mass of fully ionised helium give $M_{\mathrm{He}++} \sim 3.5 \times 10^{-6} M_{\odot}$.

We note that a correction for reddening would have increased the line luminosities, and then these values should be considered as lower limits. A total envelope mass of about $10^{-4} M_{\odot}$ is therefore indicated by the considerations of above.

\section{Conclusions}

We have analysed the observations of RR Tel taken with XMM-Newton in April 2009, which comprise high- and lowresolution X-ray spectroscopy and UV photometry.

In the UV, the comparison of the OM photometry with IUE spectra shows a gradual fading of the near UV flux, which is dominated by the recombination continuum of the nebula.

The emission in the X-ray spectral range is due to a main component arising from the hot white dwarf, and a second component due to the shocked gas within the system. While the first one had already been detected by ROSAT in 1992 and confirmed by a Swift observation in 2007 , this is the first time that the shocked-induced gas emission has been studied in detail, as it was only barely detected with ROSAT.

We compared the supersoft emission with NLTE model atmospheres and we found that it is compatible with a hot star with solar abundances, a surface gravity of $\log g=6$, and a temperature of $154 \mathrm{kK}$. Assuming a distance of $2.6 \mathrm{kpc}$, the luminosity would be $5000 L_{\odot}$. Following a different approach, that normalises the model atmospheres to the ORFEUS flux at $1100 \AA$, we arrive at a similar luminosity: $4600 L_{\odot}$ for a temperature of $150 \mathrm{kK}$.

Two plasma components are needed to explain the observed $\mathrm{X}$-ray spectrum: one with $k T=0.04 \mathrm{keV}$, an emission measure of E.M. $=10^{59} \mathrm{~cm}^{3}$, which is absorbed by a hydrogen column density of $N_{\mathrm{H}}=2.2 \times 10^{21} \mathrm{~cm}^{-2}$, and a second one with $k T=$ $0.11 \mathrm{keV}$, E.M. $=7 \times 10^{56} \mathrm{~cm}^{3}$, and $N_{\mathrm{H}}=4.2 \times 10^{21} \mathrm{~cm}^{-2}$.

Further information on the physical properties of the gas are provided by the high-resolution RGS spectra. Only a few emission lines are detected, but the He-like triplets of O VII and $\mathrm{N}$ VI clearly show that the X-ray emitting gas is collisionally ionised. The temperatures determined from the relative intensities of the lines in these triplets fully agree with those derived from the EPIC low-resolution spectra: $0.05 \mathrm{keV}$ from N VI and $0.15 \mathrm{keV}$ from $\mathrm{O}$ VII. The intercombination line is extremely weak in both triplets, thus allowing us to give only an upper limit to the electron density: $7 \times 10^{8}$ and $2 \times 10^{9} \mathrm{~cm}^{-3}$ from O VII and N VI, respectively.

Previous studies had failed to find spectroscopic signatures of the wind of the hot star. We have re-examined the existing STIS spectrum and found weak blueshifted absorptions in the N V and C IV UV doublets. The upper limit to the temperature of the gas in the shock, which was derived from the terminal velocity of the absorption lines $\left(2.9 \times 10^{6} \mathrm{~K}, 0.25 \mathrm{keV}\right)$, is fully compatible with the values obtained from the X-ray data.

The absence of typical P Cygni profiles indicates that the wind geometry is far from being spherically symmetric. The wind is not in the form of a continuous outflow of gas but likely in the form of a discontinuous, inhomogeneous ejection.

XMM-Newton observations have confirmed the presence and persistence of burning activity on the surface of the hot component for more than 65 years after outburst, as evidenced by the supersoft and hard components. To explain this long duration, 
TNR models for the outburst necessarily require the presence of a low-mass white dwarf, which they associate with the presence a rather massive envelope and with outflow at moderate velocities. Direct and circumstantial evidences, based on STIS observations and historical data (e.g. Thackeray 1977), seem to confirm this picture.

Acknowledgements. This work is based on observations obtained with $X M M-N e w t o n$, an ESA science mission with instruments and contributions directly funded by ESA Member States and NASA, and on INES data from the IUE satellite. Some of the data presented in this paper were obtained from the Mikulski Archive for Space Telescopes (MAST). STScI is operated by the Association of Universities for Research in Astronomy, Inc., under NASA contract NAS5-26555. Support for MAST for non-HST data is provided by the NASA Office of Space Science via grant NNX09AF08G and by other grants and contracts. We acknowledge with thanks the variable star observations from the AAVSO International Database contributed by observers worldwide and used in this research. We are grateful to the referee, Dr. P. Young, for his interesting remarks, which have stimulated a more detailed discussion of some of the points reported in the paper.

\section{References}

Bohlin, R., Savage, B. D., \& Drake, J. 1978, ApJ, 224, 132

González-Riestra, R., Cassatella, A., \& Selvelli, P. 2012, Mem. Soc. Astron. It., 83,806

Harper, G. M., Jordan, C., Judge, P. G., Robinson, R. D., et al. 1999, MNRAS, 303, L41

Hayes, M. A., \& Nussbaumer, H. 1996, A\&A, 161, 287

Hernanz, M. 2012, Mem. Soc. Astron. It., 83, 787

Jordan, S., Mürset, U., \& Werner, K. 1994, A\&A, 283, 475

Kaper, L., \& Henrichs, H. F. 1994, Ap\&SS, 221, 115

Krautter, J., Appenzeller, I., Mandel, H., et al. 1998, in UV Astronomy Beyond the IUE Final Archive, ESA SP, 413, 347
Kwok, S., \& Lehay, D. A. 1984, ApJ, 283, 675

Lamers, H. J. G. L. M., Stalio, R., \& Kondo, Y. 1978, ApJ, 223, 207

Landi, E., Del Zanna, G., Young, P., Dere, K., \& Mason, H. 2012, ApJ, 744, 99

Mauche, C. W. 1991, ApJ, 373, 624

Mikolajewska, J., Kenyon, S. J., Mikolajewski, M., Garcia, M. R., \& Polidan, R. S. 1995, AJ, 109, 1289

Mürset, U., \& Nussbaumer, H. 1994, A\&A, 282, 586

Mürset, U., Nussbaumer, H., Schmid, H. M., \& Vogel, M. 1991 A\&A, 248, 458

Múrset, U., Wolff, B., \& Jordan, S. 1997, A\&A, 319, 201

Ness, J. U., Starrfield, S., Osborne, J. P., Page, K., \& Schwarz, G. 2007, CBET, 1134

Nussbaumer, H. 1998, in Ultraviolet Astrophysics Beyond the IUE Final Archive, ESA SP, 413, 333

Nussbaumer, H., \& Dumm, T. 1997, A\&A, 323, 387

Nussbaumer, H., \& Walder, R. 1993, A\&A, 278, 209

Orio, M. 2012, Bull. Astron. Soc. Ind., 40, 333

Penston, M. V., Benvenuti, P., Cassatella, A., et al. 1983, MNRAS, 202, 833

Porquet, D., \& Dubau, J. 2000, A\&AS, 143, 495

Porquet, D., Mewe, R., Dubau, J., Raasen, A. J. J., \& Kaastra, J. S. 2001, A\&A, 376,1113

Porquet, D., Dubau, J., \& Grosso, N. 2010, Space Sci. Rev., 157, 103

Rauch, T. 2003, A\&A, 403, 709

Sekeráš, M., \& Skopal, A. 2012, MNRAS, 427, 979

Selvelli, P., \& Bonifacio, P. 2000, A\&A, 364, L1

Selvelli, P., \& Friedjung, M. 2003, A\&A, 401, 297

Selvelli, P., Beeckmans, F., Macchetto, F., et al. 1979, Mem. Soc. Astron. It., 50, 215

Selvelli, P., Danziger, J., \& Bonifacio, P. 2007, A\&A, 464, 71

Thackeray, A. D. 1977, MmRAS, 83, 1

Whitelock, P. 1988, in The Symbiotic Phenomenon, ASSL, 145, 47

Yaron, O., Prialnik D., Shara, M., \& Kovetz, M. 2005, ApJ, 623, 398

Young, P. R. 2012, ApJ, 749, 1

Young, P. R., Feldman, U., \& Lobel, A. 2011, ApJS, 196, 23 